\title{
Effet de différents niveaux de fertilisation azotée et du stade phénologique sur la croissance et le rendement de Brachiaria ruziziensis dans l'Ouest-Cameroun
}

\author{
Fernand TENDONKENG ${ }^{1}$, Benoît BOUKILA ${ }^{2}$, Etienne T. PAMO ${ }^{1 *}$, Arsène V. \\ MBOKO $^{2}$, Férence N. E. MATUMUINI ${ }^{2}$ et Bienvenu F. ZOGANG ${ }^{1}$ \\ ${ }^{1}$ Laboratoire de Nutrition Animale, Département des Productions Animales, FASA, \\ Université de Dschang, B.P. 222 Dschang, Cameroun. \\ ${ }^{2}$ Institut National Supérieur d'Agronomie et de Biotechnologie (INSAB), Université des Sciences \\ et Techniques de Masuku, B.P. 941. Gabon. \\ *Auteur correspondant, E-mail: ftendonkeng@yahoo.fr; pamo_te@yahoo.fr
}

\section{RESUME}

L'étude de l'effet de différents niveaux de fertilisation azotée et du stade phénologique sur la croissance et la production de biomasse de Brachiaria ruziziensis a été réalisée à l'Université de Dschang en 2008. Un dispositif factoriel comparant six doses d'azote sous forme d'urée $(0 ; 50 ; 100 ; 150 ; 200$ et $250 \mathrm{~kg}$ d'urée/ha correspondant respectivement à $0 ; 23,1 ; 46,2 ; 69,4 ; 92,5$ et 115,6 unité d'azote par ha) et trois stades phénologiques (montaison, floraison et après grenaison) sur des parcelles de $8 \mathrm{~m}^{2}(4 \times 2 \mathrm{~m})$ en quatre répétitions a été utilisé. A chaque stade phénologique, 120 plantes ont été prélevées par traitement pour les mesures des hauteurs et des diamètres. L'évaluation de la biomasse des tiges, des feuilles et de la plante entière s'est faite sur chaque parcelle en fonction du niveau de fertilisation azotée et du stade phénologique. Les résultats de ce travail ont montré que la fertilisation azotée et le stade phénologique ont influencé de manière très significative $(\mathrm{P}<0,01)$ la taille, le diamètre et la biomasse de $B$. ruziziensis. Le niveau de fertilisation avec 92,5 unités d'N/ha $(200 \mathrm{~kg} / \mathrm{ha})$ a permis à tous les stades phénologiques d'avoir la taille et le diamètre les plus élevés. La biomasse des tiges, feuilles et de la plante entière a en générale augmentée avec le niveau de fertilisation azotée pour atteindre la production maximale avec le niveau de fertilisation 92,5 unités d'N/ha, soit $200 \mathrm{~kg} \mathrm{~N} / \mathrm{ha}$ à la montaison $(6,11 \pm 0,87 ; 7,13 \pm 0,76$ et $13,24 \pm 0,70 \mathrm{t} \mathrm{MS} / \mathrm{h}$ a respectivement pour les feuilles, les tiges et la plante entière) et à la floraison $(15,54 \pm 1,96 ; 8,41 \pm 1,23$ et 23,96 $\pm 2,22 \mathrm{t}$ MS/ha respectivement pour les feuilles, les tiges et la plante entière). Indépendamment de la fertilisation, la biomasse des tiges et de la plante entière de $B$. ruziziensis a augmenté significativement $(\mathrm{p}<0,01)$ avec le stade phénologique. Aucune interaction significative $(p<0,05)$ n'a été observée entre les différents niveaux de fertilisation et les stades phénologiques. Cette étude montre que la fertilisation avec 92,5 unités d'N/ha, soit $200 \mathrm{~kg} \mathrm{~N} / \mathrm{ha}$ permet d'avoir des plantes de $B$. ruziziensis avec la taille, le diamètre et la biomasse la plus élevée, et constitue ainsi le niveau maximal de fertilisation pour cette plante dans ces latitudes.

(C) 2009 International Formulae Group. All rights reserved.

Mots clés : $B$. ruziziensis, taille, diamètre, fertilisation azotée, biomasse.

\section{INTRODUCTION}

La nécessité de nourrir une population sans cesse croissante dans les pays en voie de développement pousse de plus en plus les agriculteurs à étendre les surfaces cultivables au détriment des espaces pastorales, ce qui a pour conséquence le développement des conflits pour l'utilisation de l'espace rural entre agriculteurs et éleveurs (Boutrais, 1995; Asongwed-Awa et Njoya, 2002). Cette situation conduit au rétrécissement des parcours naturels (Pamo et al., 2001; Pamo et al., 2006a), à les repousser vers les zones marginales et aux difficultés de satisfaire les besoins nutritionnels des animaux (Pamo et al., 2006a; Pamo et al., 2006b). 
Une amélioration de la productivité animale peut passer non seulement par la maîtrise des systèmes de production, mais également par l'introduction et l'intensification de la production des espèces fourragères à haut rendement. Parmi les nombreuses espèces fourragères introduites au Cameroun, le Brachiaria ruziziensis qui est une graminée pérenne, présente les avantages de mieux s'adapter dans les différentes régions, d'avoir une bonne valeur nutritive et d'être bien appétée par les animaux (Pamo et Yonkeu, 1987; Pamo et al., 2007). La culture de cette graminée et surtout l'intensification de son utilisation peuvent constituer un palliatif au rétrécissement des parcours cidessus évoqués. Les principaux facteurs influençant sa croissance, son rendement et sa valeur nutritive sont le climat, le sol et le mode d'exploitation. B. ruziziensis est proposé pour être utilisé avec profit non seulement dans l'amélioration des pâturages naturels mais également en fourrage vert ou conservé (Cook et al., 2005). Sans fertilisation, n'importe quelle forme d'exploitation des plantes fourragères conduit à la diminution du stock en nutriment du sol en général et d'azote en particulier surtout quand il s'agit des graminées tropicales (Obulbiga et KaboréZoungrana, 2007). Différentes études ont montré que la fertilisation azotée accélère la croissance des plantes, étale la production fourragère dans le temps et entraîne une importante augmentation de la biomasse (Pamo, 1991; Lhoste et al., 1993; MorotGaudry, 1997; Peyraud et Astigarraga, 1998; Lawlor et al., 2001). Si quelques travaux ont été menés sur l'effet de la fertilisation sur le rendement de $B$. ruziziensis au Cameroun, aucune étude n'a été réalisée ni sur la détermination du niveau optimal de fumure azotée, ni sur son effet sur sa croissance surtout dans les zones d'altitude.

L'objectif de cette étude est donc de déterminer l'effet de différents niveaux de fumure azotée sur la croissance et le rendement de $B$. ruziziensis en fonction des stades phénologiques. De telles informations sont indispensables pour la formulation et la mise en place de meilleures stratégies de gestion de l'espèce.

\section{MATERIEL ET METHODES \\ Zone d'étude}

L'étude a été conduite à la Ferme d'Application et de Recherche (FAR) de l'Université de Dschang entre mars et novembre 2008. La FAR est située à $05^{\circ} 20^{\prime}$ latitude Nord et $10^{\circ} 03^{\prime}$ longitude Est et à une altitude moyenne de $1410 \mathrm{~m}$. Le climat de la région est équatorial de type Camerounien, modifié par l'altitude. Les températures oscillent entre $10{ }^{\circ} \mathrm{C}$ (juillet - août) et $25^{\circ} \mathrm{C}$ (février) avec une insolation annuelle de 1800 heures et une humidité relative variant entre $40-97 \%$. Les précipitations varient entre 1500 et $2000 \mathrm{~mm}$ par an. La saison sèche va de mi-novembre à mi-mars et la saison des pluies de mi-mars à mi-novembre correspondant à la période de culture.

La topographie du site expérimental est légèrement pentue, sur oxisol de profondeur élevée (plus de $5 \mathrm{~m}$ ) et sous pression humaine à travers la pratique des cultures vivrières annuelles. La végétation originelle de cette région est une savane arbustive avec par endroit des forêts galeries.

\section{Dispositif expérimental}

Un dispositif factoriel comparant six doses d'azote sous forme d'urée $(0 ; 50 ; 100$; $150 ; 200$ et $250 \mathrm{~kg}$ d'urée/ha correspondant respectivement à $0 ; 23,1 ; 46,2 ; 69,4 ; 92,5$ et 115,6 unités d'azote par ha) et trois stades phénologiques (montaison, floraison et après grenaison) sur des parcelles de $8 \mathrm{~m}^{2}$ (4 x $\left.2 \mathrm{~m}\right)$ en quatre répétitions, soit un total de 72 parcelles expérimentales a été utilisé. Les échantillons du sol ont été prélevés sur le site expérimental dans l'horizon $0-20 \mathrm{~cm}$ avant la préparation du sol et la mise en place des éclats de souche; l'analyse a été effectuée au Laboratoire d'Analyse des Sols et de Chimie de l'Environnement (LANASCE) suivant la méthode décrite par Pauwel et al. (1992). Il ressort de cette analyse que ce sol est limoneux et bien drainé. C'est un sol moyennement acide $(5,4<\mathrm{pH}$-eau<6,0) et ayant une acidité d'échange faible, ce qui réduit les risques de toxicité due à un excès d'aluminium et de manganèse. Les teneurs en azote total sont comprises entre $3,5 \mathrm{~g} / \mathrm{kg}$ et 3,7 $\mathrm{g} / \mathrm{kg}$ de sol, ce qui est suffisant pour l'agriculture traditionnelle, mais nécessite un complément azoté pour une agriculture intensive. Le taux de matière organique est 
élevé avec un ratio $\mathrm{C} / \mathrm{N}$ moyen de 13,5 ; ce qui lui confère une capacité de rétention en cation plus grande et lui permet de supporter une forte dose de fertilisation. Pour ce qui est des bases échangeables, le sol contient en quantité suffisante du calcium, du magnésium et du potassium. Les teneurs en sodium sont très faibles $(0,27 \pm 0,06 \mathrm{méq} / 100 \mathrm{~g}$ de sol $)$. Il en est de même de la teneur en phosphore $(1,69 \pm$ $0,28 \mathrm{mg} / \mathrm{kg}$ de sol), ce qui nécessite un apport de phosphate. Le complexe absorbant de ce sol présente une capacité d'échange cationique modéré (19,07 méq/100 g de sol) et est proportionnel aux taux de matière organique; ce qui permet d'envisager sans crainte une fertilisation avec des doses élevées d'engrais.

\section{Préparation du sol, mise en place des plants et fertilisation}

Le site expérimental a été labouré par un tracteur et la mise en place des parcelles a été faite manuellement. La même quantité (80 g) d'engrais phosphaté sous la forme de superphosphate triple a été appliquée sur toutes les parcelles comme engrais de fond. Des éclats de souche comportant trois plants de Brachiaria ruziziensis ont été prélevés dans le parcours de la FAR. Ces éclats de souche ont subi une réduction de la taille des racines et des feuilles, et cent cinq (105) éclats de souche ont été repiqués sur chaque parcelle à $4 \mathrm{~cm}$ de profondeur et suivant un écartement de $25 \times 25 \mathrm{~cm}$.

Deux mois après la plantation d'éclats de souche, la coupe de régularisation a été effectuée à $20 \mathrm{~cm}$ de la surface du sol, et les parcelles ont été fertilisées une seule fois.

\section{Collecte des données}

A chacun des trois stades phénologiques, trente (30) plantes étaient prélevées au hasard dans chaque répétition, pour un total de 120 plantes (30 plantes x 4 répétitions pour chaque niveau de fertilisation) par traitement pour les mesures des hauteurs et des diamètres. La hauteur des plantes était mesurée à l'aide d'un mètre ruban gradué au centimètre ; leur diamètre était mesuré à l'aide d'un pied à coulisse gradué au millimètre.

Pendant les périodes de coupe (24 parcelles fauchées pour chaque stade phénologique, soit 6 niveaux de fertilisation $\mathrm{x}$ 4 répétitions) et pour éviter les effets de bordure, $B$. ruziziensis était récolté (coupe de la touffe à $5 \mathrm{~cm} \mathrm{du} \mathrm{sol)} \mathrm{au} \mathrm{centre} \mathrm{de} \mathrm{la} \mathrm{planche}$ sur une parcelle utile d'une superficie de $2 \mathrm{~m}^{2}$ ( $2 \times 1 \mathrm{~m})$ et pesé. Un échantillon représentatif $(1 \mathrm{~kg})$ de chaque répétition par traitement a été prélevé et séché dans une étuve à $60{ }^{\circ} \mathrm{C}$ jusqu'à poids constant pour la détermination de la matière sèche et l'évaluation du rendement.

A chaque stade phénologique et chaque niveau de fertilisation, un échantillon représentatif de $1 \mathrm{~kg}$ des plantes entières, prélevés lors des mesures de biomasse ont été séparés en feuilles et tiges pour l'évaluation du poids relatif des différentes parties de la plantes. Leur proportion a été exprimée en matière sèche (Kaboré-Zoungrana, 1995).

\section{Analyse statistique}

Les données sur la hauteur, le diamètre et la biomasse produite ont été soumises à une analyse multifactorielle suivant le Model Linéaire Général (MLG). Lorsque les différences existaient entre les différents traitements, les moyennes étaient séparées par le test de Duncan au seuil de signification $1 \%$ (Steel et Torrie, 1980).

\section{RESULTATS}

Effet de différents niveaux de fertilisation sur la hauteur et le diamètre de Brachiaria ruziziensis à différent stade phénologique

Le tableau 1 présente la variation de la hauteur des plantes de Brachiaria ruziziensis en fonction de différents niveaux de fertilisation à la montaison, floraison et après grenaison. La taille de Brachiaria ruziziensis augmente avec la fertilisation jusqu'à 92,5 unités d'N/ha (200 kg N/ha) à la montaison, floraison et après grenaison. A la montaison, la taille des plantes fertilisées a été très significativement $(p<0,01)$ supérieure à celle des plantes non fertilisées. La fertilisation à 92,5 unités d'N/ha $(200 \mathrm{~kg} / \mathrm{ha})$ a permis à la montaison d'avoir des plantes ayant une taille très significativement $(\mathrm{p}<0,01)$ supérieure à celle des autres plantes. La taille des plantes fertilisées à 23,1 (50 kg N/ha), 46,2 (100 kg $\mathrm{N} / \mathrm{ha}), 69,4(150 \mathrm{~kg} \mathrm{~N} / \mathrm{ha})$ et $115,6(250 \mathrm{~kg}$ $\mathrm{N} / \mathrm{ha}$ ) unités d'N/ha a été comparable ( $p>0,05)$. Il en a été de même de la taille des plantes fertilisées à 23,1 et 115,6 unités d'N/ha. 
Tableau 1: Effet de différents niveaux de fertilisation sur la hauteur de Brachiaria ruziziensis à différents stades phénologiques.

\begin{tabular}{|c|c|c|c|}
\hline \multirow{2}{*}{$\begin{array}{l}\text { Niveau de fertilisation } \\
\text { (en unités d'azote/ha) }\end{array}$} & \multicolumn{3}{|c|}{ Stades phénologiques } \\
\hline & Montaison & Floraison & Après grenaison \\
\hline 0 & $100,47 \pm 14,12^{\mathrm{a}}$ & $155,55 \pm 19,44^{\mathrm{a}}$ & $176,72 \pm 18,29^{a}$ \\
\hline 23,1 & $112,77 \pm 15,94^{b c}$ & $158,80 \pm 23,02^{\mathrm{ab}}$ & $187,80 \pm 8,09^{b}$ \\
\hline 46,2 & $115,35 \pm 23,06^{\mathrm{c}}$ & $160,25 \pm 11,95^{\mathrm{ab}}$ & $191,37 \pm 19,89^{b c}$ \\
\hline 69,4 & $118,10 \pm 4,54^{\mathrm{c}}$ & $163,67 \pm 12,66^{\mathrm{b}}$ & $194,27 \pm 18,46^{\mathrm{bc}}$ \\
\hline 92,5 & $123,90 \pm 3,29^{\mathrm{d}}$ & $172,37 \pm 17,00^{\mathrm{c}}$ & $197,52 \pm 15,22^{\mathrm{c}}$ \\
\hline 115,6 & $108,85 \pm 14,67^{\mathrm{b}}$ & $158,25 \pm 21,23^{\mathrm{ab}}$ & $178,70 \pm 36,41^{\mathrm{a}}$ \\
\hline Moyenne & $113,24 \pm 15,98^{*}$ & $161,48 \pm 18,66^{3 * 6}$ & $187,73 \pm 22,34^{3 * 3 * 3}$ \\
\hline
\end{tabular}

a,b,c : les moyennes portant les lettres différentes dans la même colonne sont significativement différentes au seuil de $1 \%$. ***,***: les moyennes portant les nombres d'astérisques différents sur la même ligne sont significativement différentes au seuil de $1 \%$.

La taille des plantes fertilisées à $0,23,1$ et 46,2 unités d'N/ha a été comparable $(\mathrm{p}>0,05)$ à la floraison. Il en a été de même pour la taille des plantes fertilisées à 23,1 , 46,2, 69,4 et 115,6 unités d'N/ha. Par contre, la taille des plantes fertilisées à 92,50 unités d'N/ha a été significativement $(\mathrm{p}<0,01)$ plus que celle des plantes obtenues avec les autres niveaux de fertilisation.

La taille des plantes non fertilisées $(176,72 \pm 18,29 \mathrm{~mm})$ après grenaison a été comparable $(p>0,05)$ à celle des plantes fertilisées à 115,6 unités d'N/ha. Il en a été de même de la taille des plantes fertilisées à 115,6 ; 46,2 et 69,4 unités d'N/ha. Le niveau de fertilisation avec 92,5 unités d'N/ha a permis d'avoir des plantes avec une taille significativement $(\mathrm{p}<0,01)$ plus élevée que celle des plantes fertilisées à 0 ; 23,1 et 115,6 unités d'N/ha. Aucune différence significative n'a par ailleurs été observée entre la taille des plantes fertilisées à 46,2 ; 69,4 et 92,5 unités d'N/ha.

Le diamètre de Brachiaria ruziziensis en fonction de différents niveaux de fertilisation aux différents stades phénologiques est présenté dans le tableau 2. De manière générale, le diamètre des plantes a augmenté avec le niveau de fertilisation jusqu'à 92,5 unités d'N/ha avant de diminuer ensuite avec le niveau de fertilisation 115,6 unités d'N/ha. A la montaison, la fertilisation a permis d'obtenir des plantes de diamètre significativement $(\mathrm{p}<0,01)$ plus élevé que celle des plantes non fertilisées. Le diamètre des plantes fertilisées à 23,1 et 69,4 unités d'N/ha a été comparable. Les plantes fertilisées avec 92,5 unités d'N/ha avaient un diamètre significativement supérieur à celui des autres plantes.

A la floraison, le diamètre des plantes fertilisées a été significativement $(p<0,01)$ supérieur à celui des plantes non fertilisées. Le diamètre des plantes fertilisées avec 23,1 et 46,2 unités d'N/ha a été comparable ( $p>0,05)$. Il en a été de même du diamètre des plantes fertilisées à l'aide de 56,2 et 69,4 unités d'N/ha.

Le diamètre des plantes fertilisées à 115,6 unités d'N/ha a été comparable ( $p>0,05)$ à celui des plantes non fertilisées après grenaison. Il en a été de même pour les plantes fertilisées avec 23,1 et 46,2 unités d'N/ha. Les plantes fertilisées avec 92,5 unités d'N/ha ont eu un diamètre significativement $(\mathrm{p}<0,01)$ supérieur à celui des autres plantes. De manière générale, le diamètre des plantes le plus élevé a été obtenu avec la fertilisation à 92,5 unités d'N/ha.

Indépendamment du niveau de fertilisation, la hauteur et le diamètre ont également augmenté avec le stade phénologique (Tableau 1 et 2 ). Le diamètre des plants de $B$. ruziziensis à la floraison et après grenaison était comparable $(\mathrm{p}>0,05)$. Il était par contre significativement $(\mathrm{p}<0,01)$ supérieure à celui des plants $B$. ruziziensis à la montaison.

Effet de différents niveaux de fertilisation sur la production de biomasse de Brachiaria ruziziensis à différent stade phénologique

L'évolution de la biomasse des tiges, des feuilles et de la plante entière de Brachiaria ruziziensis à la montaison en 
fonction de la fertilisation est présentée par la figure 1. La biomasse a augmentée de manière générale avec le niveau de fertilisation azotée pour atteindre la production maximale avec 92,5 unités d'N/ha, soit $200 \mathrm{~kg} \mathrm{~N} / \mathrm{ha}$. A ce stade phénologique, la biomasse des feuilles a été supérieure à celle des tiges, indépendamment du niveau de fertilisation azotée. La biomasse des tiges et des feuilles de $B$. ruziziensis récoltée sur la parcelle témoin a été comparable $(\mathrm{p}>0,01)$ à celle obtenue sur les parcelles fertilisées avec 23,1 et 115,6 unités d'N/ha. Il en a été de même de la biomasse des tiges et des feuilles de $B$. ruziziensis récoltée sur les parcelles fertilisées avec 23,$1 ; 46,2$ et 69,4 unités d'N/ha. La biomasse la plus élevée des tiges $(6,11 \pm 0,87$ $\mathrm{t} \mathrm{MS} / \mathrm{ha})$, des feuilles $(7,13 \pm 0,76 \mathrm{t} \mathrm{MS} / \mathrm{ha})$ et de la plantes entière $(13,24 \pm 0,70 \mathrm{t} \mathrm{MS} / \mathrm{ha})$ a été obtenue avec le niveau de fertilisation de 92,5 unités d'N/ha. Avec le niveau de fertilisation de 115,6 unités d'N/ha, on a observé une baisse de la biomasse de l'ordre de $35,84 \%$ pour les tiges, $29,73 \%$ pour les feuilles et $32,47 \%$ pour la plante entière par rapport au niveau de fertilisation de 92,5 unités d'N/ha.

La biomasse des tiges, des feuilles et de la plante entière de $B$. ruziziensis récoltée à la floraison sur les parcelles fertilisées avec 0 et 23,1 unités d'N/ha a été comparable (p>0,01) (Figure 2). Il en a été de même pour la biomasse des tiges et des feuilles de $B$. ruziziensis récoltée sur les parcelles fertilisées avec 23,$1 ; 46,2$ et 69,7 unités d'N/ha. Par contre, la production de biomasse des tiges $(15,54 \pm 1,96 \mathrm{t}$ MS/ha) et des feuilles $(8,41 \pm$
1,23 t MS/ha) des parcelles fertilisées avec 92,5 unités d'N/ha a été significativement supérieure à celle des parcelles non fertilisées. La biomasse la plus élevée des tiges (15,54 \pm $1,96 \mathrm{t}$ MS/ha), des feuilles $(8,41 \pm 1,23 \mathrm{t}$ $\mathrm{MS} / \mathrm{ha})$ et de la plantes entière $(23,96 \pm 2,22 \mathrm{t}$ $\mathrm{MS} / \mathrm{ha}$ ) a été obtenue avec le niveau de fertilisation avec 92,5 unités d'N/ha. A ce stade phénologique, la biomasse des tiges a été supérieure à celles des feuilles, indépendamment du niveau de fertilisation. On a observé une baisse de la biomasse avec le niveau de fertilisation à 115,6 unités d'N/ha de l'ordre de $24,58 \% ; 15,57 \%$ et $21,45 \%$ respectivement pour les tiges, les feuilles et la plante entière par rapport au même paramètre sur les parcelles fertilisées avec 92,5 unités d'N/ha (Figure 2).

La figure 3 présente la biomasse des tiges, des feuilles et de la plante entière de $B$. ruziziensis en fonction de la fertilisation après grenaison. La biomasse des tiges à ce stade phénologique a représenté plus du double de celle des feuilles. Celles des tiges, des feuilles et de la plante entière de $B$. ruziziensis récoltées sur les parcelles fertilisées avec 0 et 23,1 unités d'N/ha a été comparables. Il en a été de même de la biomasse des tiges, des feuilles et de la plante entière de $B$. ruziziensis récoltées sur les parcelles fertilisées avec 46,2; 69,4 et 92,5 unités d'N/ha. Le niveau de fertilisation avec 92,5 unités d'N/ha a permis d'obtenir la biomasse des tiges $(17,36 \pm 0,84 \mathrm{t}$ MS/ha), des feuilles $(6,28 \pm 1,48 \mathrm{t} \mathrm{MS/ha)}$ et de la plante entière $(23,65 \pm 1,75 \mathrm{t} \mathrm{MS} / \mathrm{ha})$ la plus élevée après grenaison. Par contre, la biomasse des feuilles la plus élevée à ce

Tableau 2 : Effet de différents niveaux de fertilisation sur le diamètre de Brachiaria ruziziensis à différents stades phénologiques.

\begin{tabular}{lccc}
\hline Niveau de fertilisation & \multicolumn{3}{c}{ Stades phénologiques } \\
\cline { 2 - 4 } (en unités d'azote/ha) & Montaison & Floraison & Après grenaison \\
\hline $\mathbf{0}$ & $0,38 \pm 0,03^{\mathrm{a}}$ & $0,41 \pm 0,03^{\mathrm{a}}$ & $0,41 \pm 0,03^{\mathrm{a}}$ \\
$\mathbf{2 3 , 1}$ & $0,46 \pm 0,07^{\mathrm{c}}$ & $0,46 \pm 0,06^{\mathrm{c}}$ & $0,47 \pm 0,07^{\mathrm{b}}$ \\
$\mathbf{4 6 , 2}$ & $0,46 \pm 0,04^{\mathrm{c}}$ & $0,47 \pm 0,05^{\mathrm{cd}}$ & $0,48 \pm 0,07^{\mathrm{bc}}$ \\
$\mathbf{6 9 , 4}$ & $0,47 \pm 0,06^{\mathrm{c}}$ & $0,49 \pm 0,07^{\mathrm{d}}$ & $0,50 \pm 0,08^{\mathrm{c}}$ \\
$\mathbf{9 2 , 5}$ & $0,52 \pm 0,06^{\mathrm{d}}$ & $0,57 \pm 0,06^{\mathrm{e}}$ & $0,58 \pm 0,06^{\mathrm{d}}$ \\
$\mathbf{1 1 5 , 6}$ & $0,42 \pm 0,07^{\mathrm{b}}$ & $0,43 \pm 0,05^{\mathrm{b}}$ & $0,43 \pm 0,05^{\mathrm{a}}$ \\
\hline Moyenne & $\mathbf{0 , 4 5} \pm \mathbf{0 , 0 7 ^ { * }}$ & $\mathbf{0 . 4 7} \pm \mathbf{0 , 0 8 ^ { * * * }}$ & $\mathbf{0 , 4 8} \pm \mathbf{0 , 0 9 ^ { * * * * }}$ \\
\hline
\end{tabular}

a,b,c,d,e : les moyennes portant les lettres différentes dans la même colonne sont significativement différentes au seuil de $1 \%$. ***,***: les moyennes portant les nombres d'astérisques différents sur la même ligne sont significativement différentes au seuil de $1 \%$. 


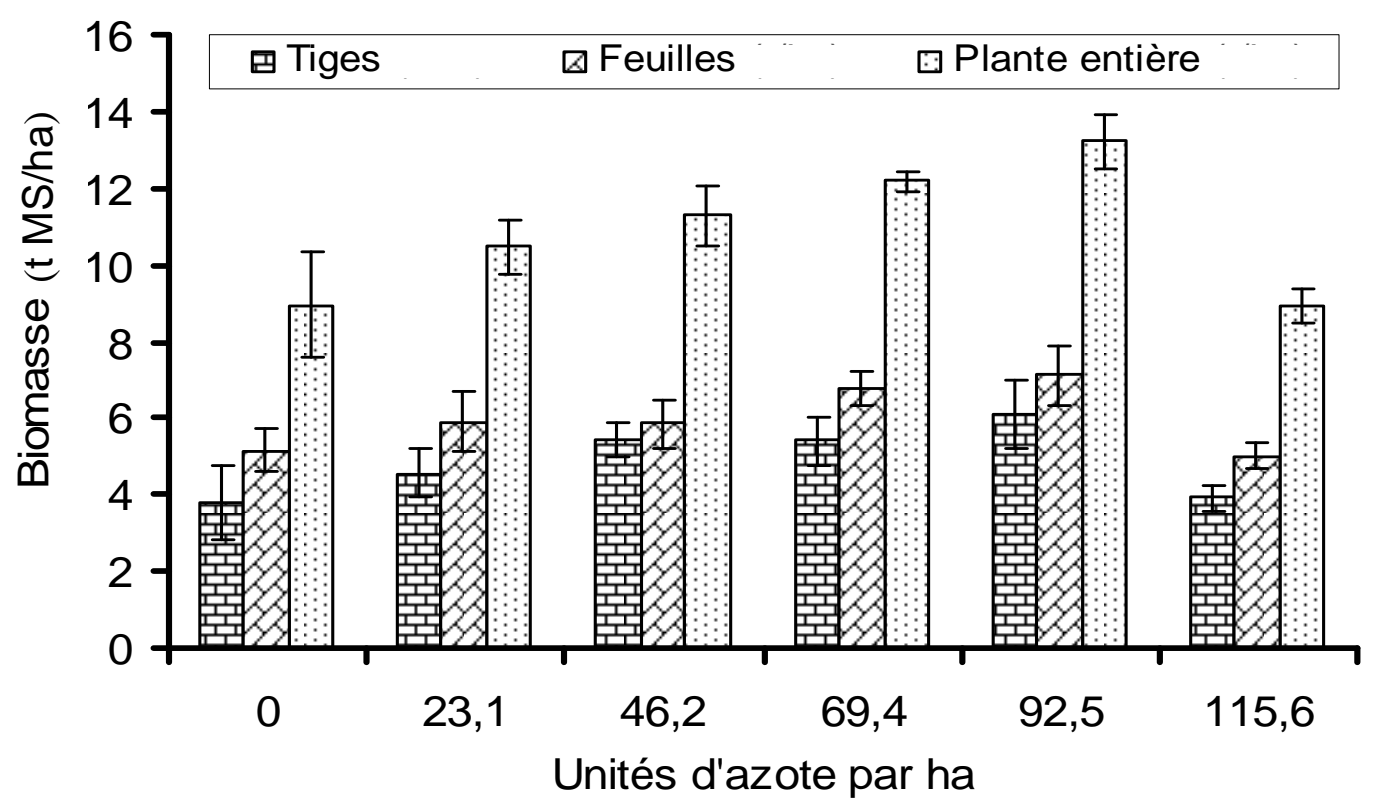

Figures 2 : Evolution de la biomasse (t MS/ha) des tiges, des feuilles et de la plante entière de Brachiaria ruziziensis à la montaison en fonction du niveau de fertilisation.

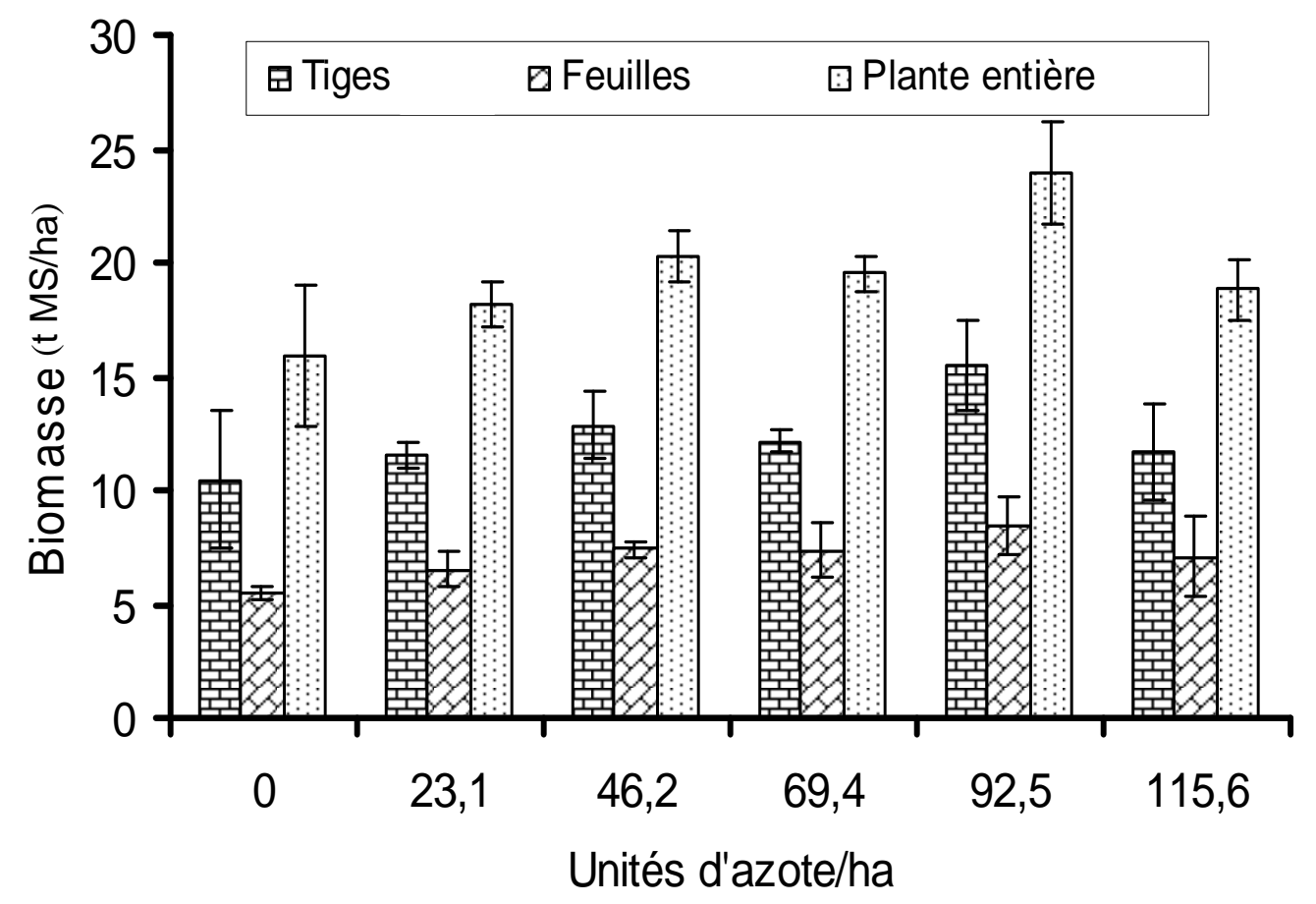

Figures 3 : Evolution de la biomasse (t MS/ha) des tiges, des feuilles et de la plante entière de Brachiaria ruziziensis à la floraison en fonction du niveau de fertilisation. 
même stade a été obtenue avec le niveau de fertilisation avec 69,4 unités d'N/ha. La fertilisation a permis de manière générale d'accroître la biomasse des tiges, des feuilles et donc de la plante entière de $B$. ruziziensis.

Ainsi, à la montaison et à la floraison, la fertilisation avec 92,5 unités d'N/ha, soit $200 \mathrm{~kg} \mathrm{~N} / \mathrm{ha}$, a permis d'obtenir la biomasse des tiges, des feuilles et de la plante entière la plus élevé. Par contre après grenaison, la fertilisation avec 69,4 unités d'N/ha a permis d'avoir la biomasse des feuilles la plus élevée bien que cette biomasse n'a pas été significativement différente $(\mathrm{p}>0,05)$ de celle obtenue avec 92,50 unités d'N/ha.

Effet du stade phénologique sur le rendement de Brachiaria ruziziensis

L'évolution de la biomasse des tiges, des feuilles et de la plante entière de Brachiaria ruziziensis en fonction du stade phénologique est présentée dans le tableau 3. Il ressort de ce tableau que la biomasse des tiges et de la plante entière a augmentée significativement $(p<0,01)$ avec le stade phénologique. La biomasse la plus élevée des tiges $(14,54 \pm 0,29 \mathrm{t} \mathrm{MS} / \mathrm{ha})$ et de la plante entière $(20,37 \pm 0,32 \mathrm{t}$ MS/ha) a été obtenue après grenaison. Par contre, la biomasse des feuilles la plus élevée a été obtenue à la floraison $(7,05 \pm 0,17 \mathrm{t} \mathrm{MS} / \mathrm{ha})$ et la plus faible après grenaison $(5,83 \pm 0,17 \mathrm{t} \mathrm{MS} / \mathrm{ha})$. Aucune différence significative $(p>0,05)$ n'a été observée entre la biomasse des feuilles de B. ruziziensis à la montaison et après grenaison. Aucune interaction significative $(\mathrm{p}<0,05)$ n'a été observée entre les différents niveaux de fertilisation et les stades phénologiques.

\section{Rapport tige/feuille}

Le rapport tige/feuille n'a pas été influencé de manière significative $(p>0,05)$ par la fertilisation azotée. Il a augmenté avec le stade phénologique (Figure 4). En effet, à la montaison, le rapport tige/feuille a varié de 0,73 à 0,93 alors qu'à la floraison et après grenaison, elle a varié de 1,64 à 1,90 et de 2,29 à 2,76 respectivement.

Tableau 3 : Evolution de la biomasse (t MS/ha) des tiges, des feuilles et de la plante entière de Brachiaria ruziziensis en fonction du stade phénologique.

\begin{tabular}{lccc}
\hline & \multicolumn{3}{c}{ Stades phénologiques } \\
\cline { 2 - 4 } & Montaison & Floraison & Après grenaison \\
\hline Tiges & $4,87 \pm 0,29^{\mathrm{a}}$ & $12,38 \pm 0,29^{\mathrm{b}}$ & $14,54 \pm 0,29^{\mathrm{c}}$ \\
Feuilles & $5,97 \pm 0,17^{\mathrm{a}}$ & $7,05 \pm 0,17^{\mathrm{b}}$ & $5,83 \pm 0,17^{\mathrm{a}}$ \\
Plante entière & $10,84 \pm 0,32^{\mathrm{a}}$ & $19,43 \pm 0,32^{\mathrm{b}}$ & $20,37 \pm 0,32^{\mathrm{c}}$ \\
\hline a,b,c $:$ les moyennes portant les lettres différentes sur la même ligne sont significativement différentes au seuil de $1 \%$.
\end{tabular}

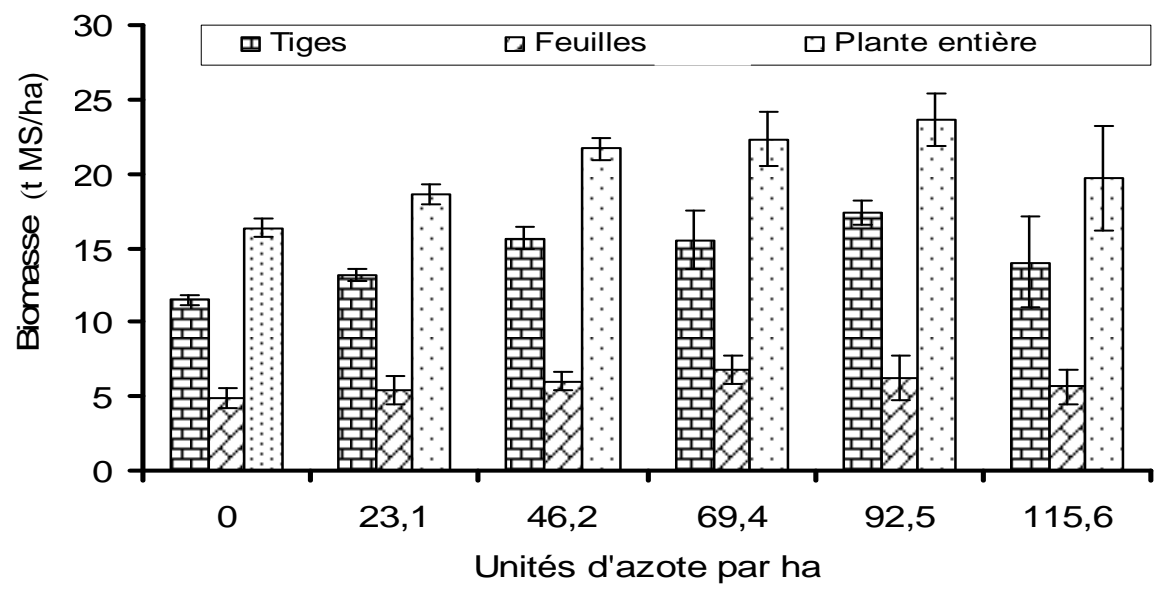

Figures 4 : Evolution de la biomasse (t MS/ha) des tiges, des feuilles et de la plante entière de Brachiaria ruziziensis après grenaison en fonction du niveau de fertilisation. 


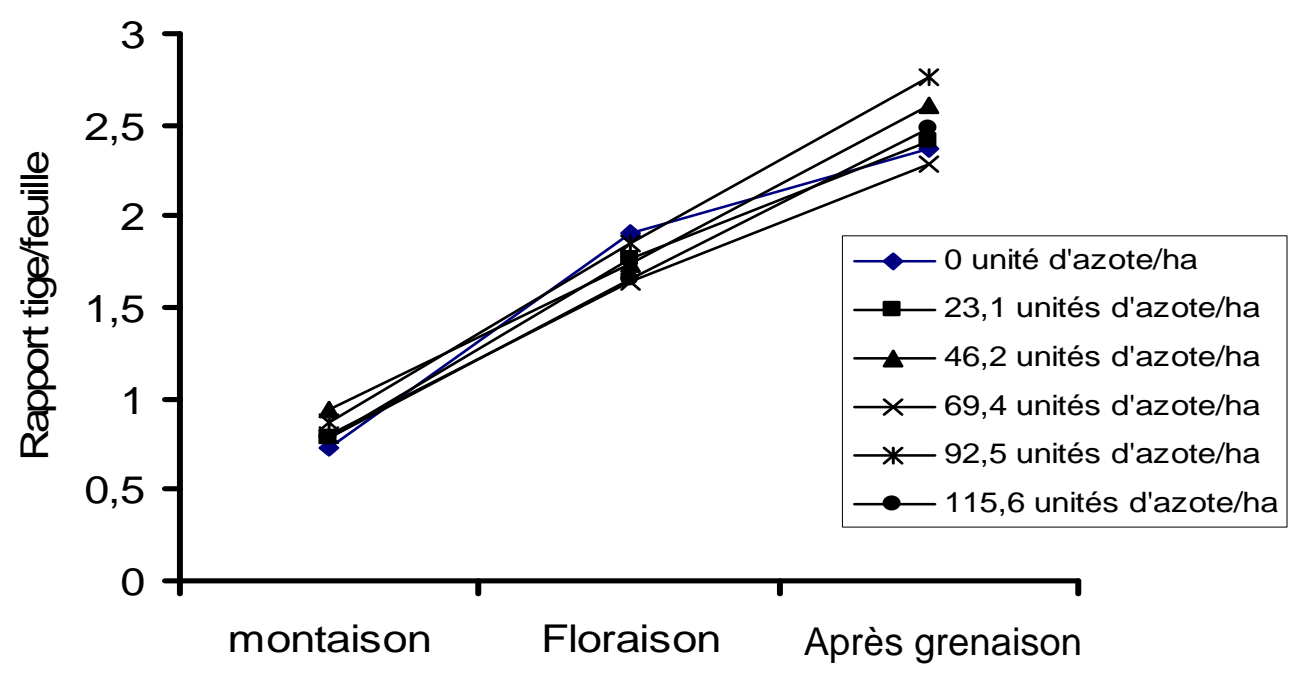

Stades phénologiques

Figures 5 : Evolution du rapport tige/feuille de Brachiaria ruziziensis en fonction du stade phénologique.

\section{DISCUSSION}

La taille et le diamètre des plantes ont augmenté avec le niveau de fertilisation azotée. A la montaison, la taille et le diamètre des plantes fertilisées étaient significativement $(\mathrm{p}<0,01)$ supérieurs à ceux des plantes non fertilisées. Par contre, à la floraison et après grenaison, la taille des plantes non fertilisées était comparable à celle des plantes fertilisées avec la plus forte dose. L'azote étant le principal facteur limitant la croissance et la production des plantes (Morot-Gaudry, 1997; Obulbiga et Kaboré-Zoungrana, 2007; Pamo et al., 2008), cette différence peut s'expliquer par le fait qu'en dépit d'une quantité suffisante d'azote dans le sol, elle ne correspondait cependant pas au maximum nécessaire pour la croissance de cette plante. Dans le cadre de cette étude, ce maximum a été atteint avec le niveau de fertilisation de 92,5 unités d'N/ha (200 kg N/ha). Nous pouvons donc penser qu'au-delà de ce niveau de fertilisation, l'azote devient toxique pour la plante. En effet, l'azote apporté sous forme d'urée s'hydrolyse rapidement pour donner le $\mathrm{NH}_{3}$ et l'ammonium $\left(\mathrm{NH}_{4}{ }^{+}\right)$. Les effets de ce dernier, nombreux et complexes lorsqu'il est en excès dans le milieu sont liés à une acidification du milieu racinaire et à des perturbations de la nutrition hydrominérale de la plante (Maurice et al., 1985; Morot-
Gaudry, 1997), ce qui expliquerait la diminution de la taille et $\mathrm{du}$ diamètre observée.

La fertilisation azotée a influencé positivement la production de biomasse de la plante entière de Brachiaria ruziziensis et de ses différentes parties. A la montaison, la proportion des feuilles était supérieure à celle des tiges, et le niveau de fertilisation avec 92,5 unités d'N/ha a induit la production de feuille la plus élevée. Par contre, à la floraison et après grenaison, la proportion de tige était supérieure à celle des feuilles, ce qui s'est traduit par un rapport tige/feuille élevé. L'augmentation de la biomasse avec le niveau de fertilisation azotée observé au cours de cet essai est en accord avec les observations de nombreux auteurs (Pamo, 1989; Pamo et Pieper, 1989; Hyo et al., 1993; Dumon et Lanuza, 1993 ; Peyraud et Astigarraga, 1998 ; Lemaire et al., 1999; Lawlor et al., 2001 ; Obulbiga et Kaboré-Zoungrana, 2007 ; Pamo et al., 2008). La variation de la biomasse obtenue dans cette étude est semblable aux observations de Bogdan (1977) et Cook et al. (2005) qui ont montré que la biomasse de $B$. ruziziensis varie de 5 à $36 \mathrm{t} \mathrm{MS} / \mathrm{h}$ e en fonction de la fertilité du sol, des précipitations et du niveau de fertilisation. En effet, la fertilisation accroît la vitesse de végétation, ce qui augmente la production pour un stade de 
croissance donné, ou réduit le délai nécessaire pour atteindre un rendement défini (Lemaire et al., 1982 ; Morot-Gaudry, 1997; Peyraud et Astigarraga, 1998; Lemaire et al., 1999). La biomasse de la plante entière obtenue à la montaison et à la floraison avec le niveau de fertilisation à 92,5 unités d'N/ha (200 kg $\mathrm{N} / \mathrm{ha}$ ) est inférieure à celle obtenue par Appadurai et Goonawardene (1973) au Sri Lanka lorsqu'il fertilisait le $B$. ruziziensis avec $224 \mathrm{~kg} \mathrm{~N} / \mathrm{ha}(22,03 \mathrm{t} \mathrm{MS} / \mathrm{ha})$ et $366 \mathrm{~kg} \mathrm{~N} / \mathrm{ha}$ (25,6 t MS/ha). Par contre, la biomasse de la plante entière obtenue avec la fertilisation à 0 et $150 \mathrm{~kg} \mathrm{~N} / \mathrm{ha}$ à la montaison $(6,7$ et $12,18 \mathrm{t}$ MS/ha) est semblable à celle obtenue avec la même espèce sur un oxisol au Brésil (6 et $12 \mathrm{t}$ MS/ha respectivement) avec les mêmes niveau de fertilisation (Vincente-Chandler et al., 1972).

Dans cette étude, nous avons observé que la biomasse augmentait avec le niveau de fertilisation jusqu'à un seuil au-delà duquel elle commençait à baisser. Ces observations concordent avec ceux de nombreux auteurs (Olsen, 1982 ; Limani et De Vienne, 2001 ; Maurice et al., 1985 ; Lawlor et al., 2001). En effet, Olsen (1982) obtient en Ouganda avec B. ruziziensis une production de biomasse de 26,5 t MS/ha avec une fertilisation maximale de $896 \mathrm{~kg} \mathrm{~N} / \mathrm{ha}$. Ce niveau maximal de fertilisation est largement supérieur à celui observé dans cette étude, qui est de 92,5 unités d'N/ha, soit $200 \mathrm{~kg} \mathrm{~N} / \mathrm{ha}$. Cette différence peut s'expliquer par la fertilité des sols et les précipitations. Par ailleurs, la baisse de rendement observée à partir d'un seuil de fertilisation est en accord avec les observations de Olsen (1982) et Limani et De Vienne (2001) qui ont montré qu'un apport d'azote à la dose qui excède les besoins de croissance potentielle de la plante ne permet plus d'augmenter le rendement fourrager. D'autre part, Maurice et al. (1985) ont montré qu'une fertilisation azotée à des doses élevées (qui excèdent les besoins de croissance potentielle de la plante) entraîne une baisse de production de la biomasse due à la toxicité de l'ion ammonium. De même, Lawlor et al. (2001) ont montré que lorsque l'apport d'azote excède les besoins de la plante, l'efficacité de son utilisation par cette dernière baisse car elle en devient saturée. L'accroissement du rendement de $B$. ruziziensis avec le stade phénologique est en accord avec celui obtenu par Lhoste et al. (1993), Daget et Godron (1995), Obulbiga et Kaboré-Zoungrana (2007) et Pamo et al. (2008). En effet, au cours de la croissance végétale, il y a augmentation de la densité de talles, développement de nouveaux bourgeons et de tiges, et une augmentation de la vitesse d'élongation des feuilles (Lemaire, 1985).

\section{Conclusion}

Il ressort de cette étude que la fertilisation azotée et le stade phénologique ont significativement influencé la taille $(\mathrm{P}<0,01)$, le diamètre $(\mathrm{P}<0,01)$ et la biomasse $(\mathrm{P}<0,01)$ de $B$. ruziziensis. La taille et le diamètre les plus élevés ont été obtenus avec le niveau de fertilisation de 92,5 unités d'N/ha, soit $200 \mathrm{~kg} \mathrm{~N} / \mathrm{ha}$. La biomasse des tiges, des feuilles et de la plante entière la plus élevée a été obtenue à la montaison et à la floraison avec le niveau de fertilisation de 92,5 unités d'N/ha, soit $200 \mathrm{~kg} \mathrm{~N} / \mathrm{ha}$. Par contre après grenaison, la biomasse des feuilles la plus élevée a été obtenue avec le niveau de fertilisation de 69,4 unités d'N/ha, soit $150 \mathrm{~kg} \mathrm{~N} / \mathrm{ha}$ tandis que celle des tiges et de la plante entière a été obtenue avec le niveau de fertilisation de 92,5 unités d'N/ha. Aucune interaction significative $(p<0,05)$ n'a été observée entre les différents niveaux de fertilisation et les stades phénologiques. Le rapport tige/feuille a augmenté avec le stade phénologique et n'a pas été influencé de manière significative $(\mathrm{p}>0,05)$ par la fertilisation azotée. Cette étude montre que la fertilisation avec 92,5 unités d'N/ha, soit 200 $\mathrm{kg} \mathrm{N} /$ ha permet d'avoir les plantes avec la taille, le diamètre et la biomasse la plus élevée d'une part, et qu'il constitue le niveau maximal de fertilisation pour cette plante dans cette zone d'autre part.

\section{REFERENCES}

Appadurai RR, Goonawardene L. 1973. Performance of three fodder grasses under high nitrogen applications in the mid-country wet zone of Sri Lanka. J. Nat. Agric. Soc., 8: 31-37.

Asongwed-Awa A, Njoya A. 2002. An integrated approach to forage seed production and supplementation of dairy cows in the semiarid region of Cameroon. Revue Elev. Méd. vét. Pays trop., 55: 269274. 
Bogdan AV. 1977. Tropical Pastures and Fodder Plants (Grasses and Legumes). London, New York, Longman; 475p.

Boutrais J. 1995. Hautes Terres d'Elevage du Cameroun (vol.1). ORSTOM 3 : Paris; 302p.

Cook BG, Pengelly BC, Brown SD, Donnelly JL, Eagles DA, Franco MA, Hanson J, Mullen BF, Partridge IJ, Peters M, Schultze-Kraft R. 2005. Tropical Forages: an Interactive Selection Tool. [CD-ROM], CSIRO, DPI\&F(Qld), CIAT and ILRI: Brisbane, Australia.

Daget P, Godron M. 1988. Pastoralisme. Troupeaux, Espaces et Sociétés. 510p.

Dumont LJC, Lanuza AF. 1993. Effect of applied and rest period on forage production and silage quality. Proceeding of the XVII International Grassland Congress, 880-881.

Hyo WL, Mu HJ, Chang HK. 1993. Effect of temperature, nitrogen fertiliser and cutting height on regrowth and dry matter production of orchagrass. Proceedings of the XVII International Grassland Congress 1993, 131-133.

Kaboré-Zoungrana CY. 1995. Composition chimique et valeur nutritive des herbacées et ligneux des pâturages soudaniens et les sous-produits du Burkina Faso. Thèse de Doctorat d'Etat. Université de Ouagadougou, 301p. + annexes.

Lawlor D, Lemaire G, Gastal F. 2001. Nitrogen, plant growth and crop yield. In Plant Nitrogen, Lea PJ, Morot-Gaudry JF (eds). INRA ; 343-367.

Lemaire G. 1985. Cinétique de croissance d'un peuplement de fétuque élevée pendant l'hiver et le printemps : effet des facteurs climatiques. Thèse d'Etat, Université de Caen, 96p.

Lemaire G, Pablo C, Jacque W. 1999. Ecophysiologie des plantes fourragères tropicales. In Cultures Fourragères Tropicales, Roberge G, Toutain (eds). CIRAD ; $19-51$.

Lemaire G, Salette J, Laissus R. 1982. Analyse de la croissance d'une prairie naturelle normande au printemps II. La dynamique d'absorption de l'azote et son efficience. Fourrages, 92 : 51-65.

Lhoste P, Dolle V, Rousseau J, Soltner D. 1993. Zootechnie des Régions Chaudes: les Systèmes d'Elevage. Manuels et
Précis d'Elevage. CIRAD, Ministère de la Coopération ; 288p.

Limami A, De Vienne D. 2001. Natural Genetic Variability in nitrogen Metabolism. In Plant Nitrogen, Lea PJ, Morot, Gaudry JF (eds). INRA; 369-378.

Maurice EH, Robert FB, Darrel SM. 1985. Forages: The Science of Grassland Agriculture. Iowa State University Press: Arnes, Iowa, USA; 318-325.

Morot-GaudryJ-F. 1997. Assimilation de l'Azote chez les Plantes: Aspects Physiologique, Biochimique et Moléculaire. Edition INRA ; 422p.

Obulbiga MF, Kaboré-Zoungrana CY. 2007. Influence de la fumure azotée et du rythme d'exploitation sur la production de matière sèche et la valeur alimentaire de Andropogo gayanus kunth au Burkina Faso. Tropicultura, 2007, 25(3): 161167.

Olsen FJ. 1982. Effect of large application of nitrogen fertilizer on the production and protein contents of four tropical grasses in Uganda. Tropical Agriculture, 49: 251260.

Pamo TE, Assontia GD, Njehoya C. 2001. Comparative growth performance of West African dwarf goat supplemented with Calliandra calothyrsus, Leucaena leucocephala, or cotton seed cake in West Cameroon. XIX International Grassland Congress. Brasil, 713-714.

Pamo TE, Boukila B, Meduke CN, Tendonkeng F. 2008. Effect of nitrogen fertilisation and cutting frequency on the yield and regrowth of Panicum maximum Jacq in West Cameroon. In: Xie Haining and Huang Jiehua (Eds). XXI International Grassland Congress / VIII International Rangeland Congress Hohhot, China, $29^{\text {th }}$ June $-5^{\text {th }}$ July 2008 , 354.

Pamo TE, Tendonkeng F. Kana JR, Boukila B. Nanda AS. 2006b. Effect Calliandra calothyrsus and Leucaena leucocephala supplementary feeding goat production in Cameroon. Small Ruminant Research, 65 : 31-37.

Pamo TE, Fonteh FA, Tendonkeng F, Kana JR, Djaga PJ, Fomewang II G. 2006a. Influence of supplementary feeding of multipurpose leguminous tree leaves on kid growth and milk production of the 
West African Dwarf goats. Small Ruminant Research, 63: 142-149.

Pamo TE. 1989. Rangeland response to low levels of nitrogen fertilization and cutting intensities on the Adamawa plateauCameroon. Rev. Elev. Méd Vété. Pays Trop., 42(4): 591-598.

Pamo TE. 1991. Réponse de Brachiaria ruziziensis Germain et Evard à la fertilisation azotée et à différents rythmes d'exploitation en Adamaoua, Cameroun. Rev. Elev. Méd. Vét. Pays Trop., 44(3): $373-380$.

Pamo TE, Boukila B, Fonteh FA, Tendonkeng F, Kana JR, Nanda AS. 2007. Nutritive values of some basic grasses and leguminous tree foliage of the Central region of Africa. Animal Feed Science and Technology, 135: 273-282.

Pamo TE, Pieper RD. 1989. Effect of nitrogen fertilization in combination with potassium and phosphorus and cutting frequency on the yield of Brachiaria ruziziensis in Adamawa plateau, Cameroon. XVI International Grassland congress, 4-11 october 1989, Nice France. Versailles, The French Grassland society, 1989, 111-112.

Pamo TE, Yonkeu S. 1987. Comportement de quelques espèces fourragères dans les bas-fonds $\mathrm{du}$ ranch SODEPA-Faro,
Adamaoua, Cameroun. Actes du séminaire régional sur les fourrages et l'alimentation des ruminants, 16-20 novembre 1987. N'Gaoundéré Cameroun. Maison-Alfort, IEMVT, Yaoundé, MESRES. (Etudes et Synthèse de l'IEMVT n $\left.{ }^{\circ} 30\right)$, 413-425.

Pauwels JM, Van Ranst E, Verloo M, Mvondo ze A. 1992. Méthode d'Analyse de Sols et de Plantes, Gestion de Stock de Verrerie et de Produits Chimiques. Manuel de Laboratoire de Pédologie. Publication Agricoles ; 286p.

Peyraud JL, Astigarraga L. 1998. Review of the effect of nitrogen fertilization on the chemical composition, intake, digestion and nutritive value of fresh herbage: consequences on animal nutrition and $\mathrm{N}$ balance. Animal Feed Science and Technology, 72: 235-259.

Steel RG, Torrie JH. 1980. Principles and Procedures of Statistics. McGraw Hill Book C: New York; 633p.

Vincente-Chandler J, Silva S, Albrunia F, Rodriguez JA. 1972. Effect of two cutting heights, four harvest intervals and five nitrogen rates on yield and composition of Congo grass under humid tropical conditions. Puerto Rico Journal of Agriculture, 56: 280-291. 